\title{
Identification of Novel Chemical Entities for Mirk/Dyrk1B Receptor Using Molecular Modeling and Molecular Dynamic Approaches
}

\author{
Ramar Vanajothi \\ Bharathidasan University \\ Sundaresan Bhavaniramya \\ Tamil Nadu Veterinary and Animal Sciences University \\ Muthu Umadevi \\ Nehru Memorial College \\ Rajendran Vijayakumar \\ Majmaah University \\ Yaser E. Alqurashi \\ Majmaah University \\ Kumpati Premkumar ( $\sim$ premslab@gmail.com ) \\ Bharathidasan University
}

\section{Research Article}

Keywords: Mirk, homology modeling, virtual screening, molecular dynamics simulation.

Posted Date: December 16th, 2021

DOI: https://doi.org/10.21203/rs.3.rs-1141061/v1

License: (c) (i) This work is licensed under a Creative Commons Attribution 4.0 International License. Read Full License 


\section{Abstract}

Cancer is a major health problem worldwide and one of the leading death-causing diseases. Mirk (Minibrain-related kinase is a member of the dual-specificity tyrosine-phosphorylation-regulated kinase (Dyrk) family that is highly upregulated in various solid tumors and mediates cell survival including lung cancer. Mirk effectively increases the expression of a series of antioxidant genes, which scavenge the reactive oxygen species and stabilize the p $27^{\mathrm{kip} 1}$ that maintain the viability of the quiescent cancer cell and also mediates the cell cycle and survival of cancer cells by influencing the MAPK/ERK signaling pathway. Hence, Mirk acts as a novel therapeutic target for cancer prevention. Owing to the unavailability of the three-dimensional structure of Mirk, in the present study, we have modeled the 3D structure of Mirk, based on the crystal structure of Dyrk1a as a template, and subsequently used it as a target for virtual screening and molecular docking against a small molecule database. Based on the visual inspection, four best hits such as Chembridge_ID 7768949, 7771055, 7758866, and 7764195 have high binding affinity, good docking score, and pharmacokinetic properties were shortlisted. Further, the dynamic stability of lead molecules with modeled Mirk/Dyrk1B was evaluated using 10 ns molecular dynamics simulation approach. The four hit molecules exhibited good and stable binding complex in the binding pocket of the target protein. Collectively the finding of this study suggested that the identified molecules may serve as potential effective anticancer inhibitors for cancer prevention.

\section{Introduction}

Cancer is generally characterized by uncontrolled proliferation and impaired apoptosis. Mirk/Dyrk1B is a member of the dual-specificity tyrosine-phosphorylation-regulated kinase (Dyrk) family. It contains four different isoforms such as Dyrk1B, Dyrk2, Dyrk3, and Dyrk4. Several reports suggested that Dyrk1B plays a crucial role in cancer biology and muscle differentiation [1-4]. It has the ability to auto-phosphorylate serine/threonine hence it is also characterized as dual function kinase. The low level of Mirk/Dyrk1B was noticed in normal tissues and it is highly expressed in normal skeletal muscles and several solid tumors including lung cancer, where it mediates cell survival and differentiation. It elevated the expression of the antioxidant gene in cancer cells and it reduces the toxic ROS generation by which it mediates the survival in these cells. In addition, it has the unusual property of being most active in quiescent cancer cells $[4,5]$. Genetic alteration including mutations, translocations, and amplifications can lead to various pathological diseases including cancer. Amplicons are maintained in cancer cells when the amplified genes provide selective growth. A recent study reported that Mirk is one of the 16 genes within a consistently amplified $660 \mathrm{~kb}$ sub-region of the 19q13 amplicon in pancreatic cancer [6], and ovarian cancer [7]. These amplicons are maintained in most of the cancer cells and provide a selective growth or survival advantage Dryk1B is the small protein kinase protein that may be closely related to cancer the expression of Dyrk1B is substantially increases during cell division to cell differentiation, the mode of control on these processes are poorly understood. Number of evidence has been reported in past decades to discern the impact of knockdown Mirk/Dyrk1B on apoptosis and how it increases the sensitivity of various cancer cells [3]. Gao et al., [4] reported that Mirk overexpression promotes the survival of lung cancer cells. It also promotes the survival of differentiating myoblast by phosphorylating the CDK inhibitors p21, which leads to the accumulation of p21 in the cytoplasm and act as an antiapoptotic signaling molecule [8], and also mediate the differentiation of normal tissues like neuronal cells (Dyrk1A), skeletal muscle (Mirk/dyrk1B), erythropoietic cells (Dryk3) [9-12, 8]. Therefore, Mirk/Dyrk1B acts as a novel therapeutic target for cancer prevention. Generally, the Mirk activity is not increased by a mutation in cancer cells, however, it abundantly increases several-fold when the cells leave the cell and become quiescent and also increases when exposure to chemotherapeutic drugs like 5-Fu and cisplatin [12-14]. During the translation process, the Dyrk kinases can autophosphorylate on tyrosine and can phosphorylate the other substrate like serine and threonine; hence they are classified as dual function kinases. The Mirk/Dyrk1B expression level was low in normal colon and lung cancer tissues [15]. Several studies have been reported that the Mirk has been demonstrated to play a crucial role in regulatory effect on mortality, and transcription, and cell cycle $[9,11]$. The above data on tumor biology studies indicated that the high expression of Mirk solid tumors can lead the tumor cell survival in different types of human cancer including colon, lung, 
pancreatic ductal adenocarcinoma cell and HeLa cervical carcinoma cells $[11,15,16]$. Knockdown studies of Mirk studies revealed that it does not cause embryonic lethality and is not essential for normal cell growth and may consider a novel and important therapeutic target [11]. Hence the present study was designed to find the potential Mirk inhibitors via a computational approach. Due unavailability of the 3-dimensional structure of Mirk, a molecular modeling approach was done to model the Mirk structure and virtual screening against the modeled structure was performed using phase module implemented in Schrodinger and molecular docking and simulation were performed to evaluate the interaction of lead molecules with active site residues and its stability respectively. In addition, the drug-likeness properties of the screened lead molecules were checked with the Qikprop module.

\section{Materials And Methods}

\section{Template selection and Sequence alignment}

The amino acid sequence of human Mirk (Accession number: Q9Y463) was retrieved from UniProtKB/Swiss-Prot database (http://ca.expasy.org) and submitted to the Pfam server for prediction of active domain. The predicted domain sequence was subjected to pair-wise alignment using NCBI-BLAST against Protein Data Bank (PDB) to find a template with a suitable identity. Based on the resolution, sequence identity, alignment score, positives, and gaps present between the template and the target sequences. The high amino acid sequence similarity between the target protein and templates suggested that 4MQ1 was selected as a suitable template. Multiple sequence alignment of Mirk protein sequence along with the homologous was generated by Clustal W.

\section{Homology modeling}

The 3D structure of human Mirk/Dyrk1B was built by homology modeling. The X-ray crystal structure of 4MQ1 was retrieved from the PDB (http://www.rcsb.org/pdb) as a template to generate the model. Molecular modeling was performed using the MODELLER 9v7 (http://salilab.org/modeller) program with default parameters. The sequence file, alignment file was executed by the modeler command prompt. About 5 models were generated for Mirk with their respective DOPE scores. The model with the least DOPE score was selected for the final validation. MODELLER uses a spatial restraint method to build up protein 3D models. The model was then energy minimized in Swiss-PDB viewer. The outcomes of the modeled structures were ranked based on an internal scoring function, and those with the least internal scores were identified and utilized for model validation.

\section{Structure evaluation}

The quality of the model was assessed by comparing predicted structure to X-ray solved structure via superposition and measurement of the C-a Root Mean Square Deviation assessment (RMSD). The predicted model and the template 3D structure were superimposed using Pymol and the RMSD between the predicted model and template was identified. The quality and validation of the final developed model with the lowest DOPE score were assessed by Structural Analysis and Verification Server (SAVES), which validates the modeled structure using the programs PROCHECK, Verify3D, and ERRAT. It evaluates the side-chain conformation and overall structure geometry of the modeled protein. Ramachandran plot was analyzed to evaluate the stereochemical stability of the predicted model were verified with various parameters including residues lying in both allowed and disallowed regions, the orientation of dihedral angles (phi and psi), and the number of glycine and proline residues obtained by PROCHECK $[17,18]$.

\section{Molecular dynamics}

All the simulations were carried out using the GROMACS simulation program (GROMACS 4.5.1) adapting the GROMOS $43 a 1$ force field $[19,20]$. The topological parameters lead of inhibitors was generated using the PRODRUG server [21]. The 
systems were solvated using a water model of a single point charge (SPC) in a dodecahedral periodic box keeping a minimum distance of $1.0 \mathrm{~A}$ between the solute and edge of the box. Then the system was neutralized by the addition of counter ions ( $\mathrm{Na}+$ and $\mathrm{Cl}-$ ), at a concentration of $0.15 \mathrm{M}$, which mimic the physiological concentration. Before the simulation, the system was energy minimized using the steepest descent minimization algorithm (ref) with a maximum of 50000 steps and maximum force was lessened to $100 \mathrm{kcal} \mathrm{mol}-1 \mathrm{~nm}-1$. Then 100 ps NVT (constant number of particles, volume, and temperature) equilibration followed by 100 ps NPT (constant number of particles, pressure, and temperature; 300 K, 1 bar; Berendsen coupling and Parrinello-Rahman method) equilibration were carried out for each system to allow the solvent to equilibrate around the solute [22, 23]. All the bond lengths of water molecules were constrained adopting the LINCS and SETILE algorithm and electrostatic and van der Waals (vdW) were applied based on the particle mesh Ewald (PME) method. MD conformations were saved every 2 ps for further analysis [24].

\section{Active site prediction}

The computationally modeled Mirk structure was further subjected to a sitemap program implemented in Schrödinger [25], to forecast the active sites of the target protein. SiteMap predicts the probable pockets or sites for ligand binding and generates information on the binding site's characteristics using novel search and analytical facilities. SiteMap calculation begins with an initial search step that generates site points on a grid surrounding the protein surface that may be suitable for binding ligands to the receptor. Contour maps are then generated, producing hydrophobic and hydrophilic maps which may guide protein-ligand docking analysis.

\section{Protein preparation}

The modeled Mirk protein was subjected to molecular docking studies. Prior to the docking, water and molecules were removed following which, hydrogen was added to carry out restrained minimization of the modeled protein by protein prep wizard implemented in Schrodinger. Bond order and partial atomic charges were assigned according to the optimized potentials for liquid simulation (OPLS)-2005 force field. The restrained minimization of the protein was performed using the default constraint of $0.30 \AA ̊$ root mean square deviations (RMSD).

\section{Virtual screening}

Chembridge database compounds were docked into the active site of the Mirk structure using the Glide High-throughput Virtual Screening (HTVS) docking module [26]. The Chembridge database contains about 1.3 million small molecules that have been filtered by Lipinski's rule of 5 to get orally active molecules. For maximum diversity of the small molecules, different tautomeric and ionization states were also generated. The modeled structure of Mirk was used as an initial model for screening. A receptor grid of $10 \AA \times 10 \AA \times 10 \AA$ was generated on predicted binding site residues of Mirk using Glide implemented in Schrodinger, LLC. After the grid generation, the hierarchical filtering protocol was performed based on different levels of the precision score function to identify potential inhibitors [27]. All the compounds were initially docked by the HTVS precision procedure followed by a standard procedure (SP), and finally the extra precision (XP) procedure. The best lead molecules were selected based on the Glide score, Glide energy, and hydrogen bond interaction [28, 29].

\section{Binding energy calculation}

The binding affinity of lead molecules was rescored by prime calculation withOPLS3/3e force field by MM/GBSA implemented in Schrodinger software using Prime program with both ligand and receptor flexibility. The binding free energy $\left(\Delta G_{\text {bind }}\right)$ of each docked pose was estimated based on the molecular mechanics $(M M)$ and Generalized Born Surface Accessible (GBSA) model. It is an acronym for a method that combines OPLS-AA (optimized Potential for Liquid Stimulation-All Atom) molecular mechanics energies (EMM), an SGB salvation model for polar salvation (GSGB), and a nonpolar salvation term (GNP) composed of the nonpolar solvent accessible surface area and van der Waals interactions [30]. Binding energy was calculated by the following formula: 
$\Delta \mathrm{E}=\mathrm{E}_{\text {complex }}-\mathrm{E}_{\text {protein }}-\mathrm{E}_{\text {ligand }}$

Where $E_{\text {complex }}$ is the energy of the protein-inhibitor complex, $E_{\text {protein }}$ is the energy of protein and $E_{\text {ligand }}$ is the energy of ligand [31, 32].

\section{ADME}

The pharmacokinetic properties of the best lead molecules were studied calculated by QikProp [33]. It predicts physically significant descriptors and pharmaceutically relevant properties of organic molecules. In the present study, QikProp properties such as MDCK cell permeability (QPPMDCK), Lipinski,s rule of five, IC50 value for blockage of HERG K+ (QplogHERG), Caco-2 cell permeability (QPPCaco), logP and human oral absorption (QP\%), aqueous solubility QplogS were predicted to qualify the overall potential of lead molecules as a drug [34].

\section{Result And Discussion}

\section{Homology modeling}

Homology modeling estimates the 3D structure of a target protein sequence by using its alignment to one or more protein templates of known structure [35]. Though, Mirk plays a crucial role in various solid tumors and acts as a potential therapeutic drug target. Still, now there is no 3D structure of Mirk protein was deposited in PDB; hence a homology model was performed to determine the 3D coordination of Mirk protein. The sequence alignment of 4MQ1 (template) and (target) and the secondary structural elements are indicated in Figure 1. Template searching against Protein Data Bank (PDB) through BlastP was performed and 4MQ1 was selected based on identity (42\%) with the lowest E-Value (3e-23). The threedimensional structure provides valuable insight into its molecular function and also enables the analysis of its interactions with suitable substrates or inhibitors [36].

On the basis of the sequence alignment, the 3D structure of the target protein was predicted using the crystal structure of the template $(4 \mathrm{M})$ by comparative modeling using a modeler. It generates five models, based on the DOPE score model-4 was selected for further analysis (Figure 2). The root means square deviation of the model relative to the template (4MQ1) $0.111 \mathrm{~A}$ was calculated using Pymol. It indicates that the modeled protein show close homology to the template.

\section{Structure evaluation}

In order to evaluate whether a model satisfies standard steric and geometric criteria and select the best homologs model, we used several verification tools for model evaluation such as PROCHEK, WHAT_CHECK, ERRAT, VERIFI_3D, and PROVE provided by the SAVS server. PROCHEK was used to perform full geometric analysis as well as the stereo-chemical quality of a protein structure by analyzing residue-by-residue geometry and overall structure geometry [37, 38]. The stereochemistry assessment of the Mirk model showed more than $92.4 \%$ residues of psi and phi angles are in the favored region of the Ramachandran plot (Figure 3 ) and $6.4 \%, 0.4 \%$ of residues belong to the generously allowed region and allowed respectively. Concerning the Ramachandran plot, it is observed that there are $0.4 \%$ residues in the disallowed region; it again indicates that the final model was more reliable and had good quality and was close to the template structure, and could be used for further studies. Figure 3B represent that the residue interaction energies; which shows the energies remain negative for almost all the residue indicating the acceptability of the model. Profile score above zero in the Verify 3D graph $[39,40]$ corresponds to the acceptable environment of the model. The ProSA [41] results in finding the potential error on the predicted model revealed the Z-score values of -4.56 against the template which indicates that protein structure is well correlated with the crystal structure of similar length and within the acceptable range.

\section{Virtual screening and docking studies}


The active site of the modeled Mirk was predicted by the sitemap program implemented in Schrodinger. The grid was generated considering the centroid of active site residues in the binding region for further virtual screening. Chembridge database was used to find potential inhibitors. The validated model of Mirk and ligands of Chembridge database were imported into Schrodinger LLC, 2018). Prior to docking ligands were prepared to expand protonation and tautomeric states of each ligand molecule at $7.0 \mathrm{pH}$ with LigPrep module implemented in maestro $[42,43]$. The high energy tautomer state or ionization state and the ligand did not follow Lipinski's rule, or the molecules with reactive functional groups are eliminated from the generated confirmation. Like ligand preparation, prior to docking the predicted Mirk model was also subjected to preprocess with Protein preparation Wizard in the maestro, where all the hydrogens were added and subsequently subjected into minimization with an OPLS-2005 force field and the impact of molecular mechanics engine [44, 45]. The minimization process restraining heavy atoms and allows free rotation of hydrogen setting with an RMSD value of $0.30 \AA$ [46]. The active site was predicted with the Sitemap module and a grid box was generated around the active site of the model [47]. Glide virtual screening, SP, (standard precision), and XP (extra precision) mode were applied to predict the potential lead molecules. The good scoring ligands were ranked based on glide score and binding energy with predicted Mirk modeled protein.

\section{In silico predicted Physico-chemical parameters}

The top-ranked compounds (table 1) were subjected to a QikProp module to examine the drug-like behavior of the compounds by evaluating their pharmacokinetic factors. All the important parameters were depicted in table 1. All the topranked molecules exhibited a molecular weight in the acceptable range. ADME properties of lead molecules play a crucial role in medicinal chemistry. The pharmacokinetic parameters will influence the drug-likeness of the lead molecules. Hence in the present study, in silico analysis of physicochemical properties of the top-ranked hits was predicted. The values of QPlogS, QPlogBB, and QPlogPo/w were noticed within the prescribed limit -6.5 to $0.5,-3.0$ to 1.2 , and -2.0 to 6.5 respectively. The value of predicted Caco-2 cell permeability of the top-ranked lead molecules was found to be very high except for the compound ID 7753623, this result suggested that the ChembrigelD: 7753623 may face some hurdles in the absorption process than other lead molecules. The value of permeability through MDCK cells of all the top-ranked compounds was found to be <500, except ChembrigelD: 7764195, the results depicted that the MDCK cells are considered to be a good mimic for the blood-brain barrier. Overall results indicated that all the top-ranked compounds are satisfied with rules with fewer violations. Additionally, prime MM-GBSA binding energy was calculated for the top two compounds based on their binding affinity towards the active site of Mirk protein. MM-GBSA score of Chembridge ID: 7768949 was -102.21 whereas the second topmost compound ID: 7771055 has shown a slightly lower MM-GBSA score of -96.04 . With these results, it can be concluded that the binding energy of both the compound shows good binding interaction and binding energy with the active site of the Mirk protein.

\section{Molecular docking}

Molecular docking studies revealed the apparent binding mode of lead molecules in the active site of the selected drug targets with maximum docking score and binding energy. The modeled Mirk was docked with Chembridge database ligand molecules to generate the binding mode and dynamic simulation was done to refine the best pose of top-ranked compounds with an allowed conformational change in the Mirk. The top-ranked compounds such as Chembridge ID; $7768949,7771055,7758866$, and 7764195 were shows good docking scores ranging from -11.361 to -10.064 . The docked poses of top-ranked compounds at the active site of modeled Mirk protein are shown in Figure 4. The detailed analysis of residues and hydrogen bond interactions of lead molecules were shown in the 2D diagram in Figure 5 and Table 2 . The binding mode analysis shows that the binding energy range of lead molecules was ranged between $-49.771 \mathrm{Kcal} / \mathrm{mol}$ to $-32.065 \mathrm{Kcal} / \mathrm{mol}$. The amino acid residues involved in both bonding and non-bonding interaction with lead molecules with Asn86, Leu83. Lys17, lle7 play a crucial role in ligand binding. Based on the modeled structure, we speculated that the identified lead molecules significantly positioned in the binding pocket of the modeled structure with good stability. 
The molecular interaction diagram (Figure 5) shows the interaction of the best two compounds such as ChembridgelD: 7768949 and ChembridgelD: 7771055 with active site residues. Chembridge ID 7768949 formed 2 hydrogen bond interactions with Leu83 via $-\mathrm{N}$ - and $-\mathrm{NH}$ - atom ( $\mathrm{N}$... H, NH...Leu83) with $2.30 \AA ̊$ distance and another hydrogen bond interaction with same residues via $-\mathrm{C}$ - and $-\mathrm{OH}$ atom $(\mathrm{C} . . . \mathrm{H}, \mathrm{OH}$...Leu83), with a distance of $1.89 \AA$ also it forms hydrogen bond interaction with Ile7 via $-\mathrm{C}$ - and $-\mathrm{OH}$ atom $(\mathrm{C} . . . \mathrm{H}, \mathrm{OH}$...lle7) with $2.01 \AA$ distance. Compound ID 7771055 forms two hydrogen bond interactions with Leu83 via $-\mathrm{N}$ - and $-\mathrm{NH}$ - atom $(\mathrm{N} . . . \mathrm{H}, \mathrm{NH}$...Leu83) and $-\mathrm{C}$ - and $-\mathrm{OH}$ atom $(\mathrm{C} . . . \mathrm{H}, \mathrm{OH} . .$. Leu83) with the distance of $2.04 \AA$ and $2.34 \AA$ respectively, also form hydrogen bond interaction with Asn 86 via - C- and -

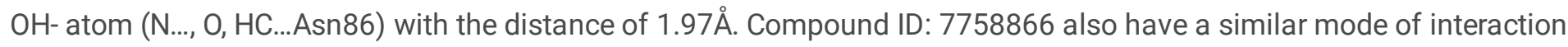
like Chembridge ID 7768949, formed 2 hydrogen bond interaction with Leu83 via - $\mathrm{N}$ - and $-\mathrm{NH}$ - atom ( $\mathrm{N}$...H, NH...Leu83) and $-\mathrm{C}$ - and $-\mathrm{OH}$ atom $(\mathrm{C} . . . \mathrm{H}, \mathrm{OH} . . . \mathrm{Leu} 83)$ with a distance of $1.94 \AA$ and $2.30 \AA$ respectively, also form hydrogen bond

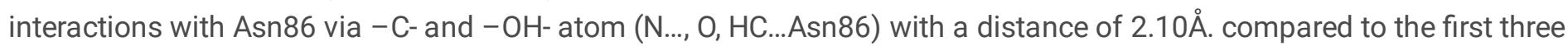
compounds, compound ID: 7764195 shows the different mode of interaction in the active site of Mirk receptor, it forms hydrogen bond interaction with Leu via $-\mathrm{N}$ - and $-\mathrm{NH}$ - atom $(\mathrm{N} . . . \mathrm{H}, \mathrm{NH} . .$. Leu83) with a distance of $2.10 \AA$ and with Lys 17 via $-\mathrm{N}$ - and $-\mathrm{NH}$ - atom (N...H, NH...Lys17) with a distance of $2.60 \AA$ and form another hydrogen bond interaction with lle7 via $-\mathrm{C}$ - and $-\mathrm{OH}$ atom $(\mathrm{C} . . . \mathrm{H}, \mathrm{OH}$...lle7) with $2.01 \AA$ distance This interaction studies revealed that hydrogen bond formations were responsible for he binding affinity of the compounds with active site residues. The molecular contact profiling suggested that virtually screened compound show the potential to inhibit the Mirk/Dyrk1B protein via action on the binding of crucial amino acid residues to distort the active binding pocket of Mirk/Dyrk1B.

\section{MD simulation}

A molecular dynamic simulation was performed using GROMACS to evaluate the structural stability of both modeled protein and complexes with top-ranked compounds. To confirm the structural stability and equilibrium state of the molecular simulation, root mean square deviation (RMSD) of backbone carbon atoms of both modeled protein and complexes with top-ranked compounds are calculated and plotted in figure 6. From the RMSD plot, it was observed that the modeled protein Mirk was significantly stable throughout the simulation time of $20 \mathrm{~ns}$ in the range of 0.14 to $0.17 \mathrm{~nm}$. Whereas the top-ranked complexes such as Chembridge ID: 7768949 and Chembridge ID: 7771055 with modeled protein show higher RMSD fluctuation arise till $5 \mathrm{~ns}$ in the range of 0.25 and slightly down in the range of 0.15 to $020 \mathrm{~ns}$ and get stable remaining simulation time (figure 7). The RMSD of a compound such as Chembridge ID: 7758866 and Chembridge ID: 7764195 shows fluctuation similar to first two top-ranked compounds and arise up to $5 \mathrm{~ns}$ in the range of 0.25 and highly fluctuate up to in the range of 0.30 to 0.35 till 20 ns simulation period (Figure 7). The RMSF of modeled protein residues lies below $0.21 \mathrm{~nm}$ (figure 8).

The binding of top-ranked compounds such as (compound 3 and 4) with modeled protein shows little fluctuation and shows RMSF value up to $0.4 \mathrm{~nm}$ and $0.5 \mathrm{~nm}$ respectively, however, the active site residues do not show high fluctuation throughout the simulation time. After binding of compounds 1 and 2, the modeled protein does not show any high fluctuations throughout the simulation time and both the compounds retain stability in the whole simulation period (Figure 7). From the RMSD and RMSF results, it depicted that the top-ranked screened compounds effectively perturb to the binding pocket of the modeled protein. In addition to the stability analysis, the protein interaction with top-ranked compounds was supervised throughout the simulation period. The hydrogen bond interaction of crucial amino acids is present in the active site of the protein-making the protein-ligand complex stable.

The molecular simulation studies indicate that the both modeled and complex system shows significant stability, and results of hydrogen bond interaction of top-ranked complexes also support this statement (Figure 8). The amino acid residues Asn86, Lue83, and Ile7 exhibited strong hydrogen bond interaction with all the top-ranked compounds revealed by docking studies also existed in molecular dynamics simulation trajectory, with stable and strong interaction in the stipulated time. Compared to compounds 1 and 2, compounds 3 and 4 showed less stable interactions with similar amino 
acid residues during the entire simulation the dark color represents the number of interactions with respective of the amino acid high, and minimal backbone fluctuation has ensued in the system.

\section{Conclusion}

Identification of potent efficient and target specific against cancer targets are of significant interest to prevent cancer or cancer treatment. Mirk is belonged to an evolutionarily conserved family that play a crucial role in cell differentiation and proliferation [48, 9], the up-regulation of mirk in human cancer is highly responsible for the maintenance of cancer cells in their quiescent state to confer chemoresistance $[49,50]$. The current study was designed to find the potential compound against the mirk receptor via the in silico approach. The Chembridge database compounds were subjected to structurebased virtual screening, which results in four lead compounds with high docking scores and binding energy. All the topranked compounds bind the similar binding mode and hydrogen bond interaction with crucial amino acids. The molecular simulation studies showed that both the modeled structure and complex system were significantly stable throughout the simulation time. Pharmacokinetics studies also supported the drug-likeness properties with an acceptable range. Overall the study concluded that the lead molecules ay act as potent inhibitors against the Mirk receptor.

\section{Declarations}

\section{Funding}

Not applicable

\section{Conflict of interest}

The authors declare no conflict of interest, financial or otherwise.

\section{Acknowledgements}

One of the authors, Ramar Vanajothi thankfully acknowledges UGC for providing the Kothari fellowship (BL/17-18/0210). Authors, Vijayakumar, and Yaser E. Alqurashi would like to thank Deanship of Scientific Research at Majmaah University.

\section{Availability of data and material}

Authors have declared that there is no any data material related to this study.

\section{Code availability}

Data sharing not applicable to this article as no datasets were generated or analysed during the current study.

\section{Authors' contributions}

R.V and S.B. conceived of the presented idea. R.V developed the theory and performed the computations. M.U, Y.E.A and R.V.K. verified the methods and corrected the manuscript. K.P. to investigate and supervised the findings of this work. All authors discussed the results and contributed to the final manuscript.

\section{References}

1. E. Friedman. Cancers (Basel). 2010,14,1492-1512.

2. K. Jin, S. Park, D. Z. Ewton. Cancer Res., 2007, 67, 7247-7255.

3. E. Friedman. J Cell Biochem., 2007, 102, 247-249.

4. G. Gao, Z. Zheng, B. Rawal. Cancer Biol Ther., 2009; 8, 1671-1679 
5. X. Deng, J. Hu, M. J. Cunningham, E. Friedman. Genes and Cancer., 2014, 5, 201-211.

6. R. Kuuselo, K. Savinainen, D. O. Azorsa. Cancer. Res., 2007, 67, 1943-9.

7. F.H. Thompson, M.A. Nelson, J.M. Trent, X.Y. Guan, Y. Liu, J. M. Yang, J. Emerson, L. Adair, J. Wymer, C. Balfour, K. Massey, R. Weinstein, D.S. Alberts, R. Taetle. Cancer Genet Cytogenet., 1996, 87, 55-62.

8. S. E Mercer, D. Z. Ewton, S. Shah, A. Naqvi, Friedman E. Cancer Res. 2006, 66, 5143-50

9. S. E. Mercer, E. Friedman. Cell Biochem Biophys. 2006,45, 303-315.

10. S. Leder, Y. Weber, X. Altafaj, X. Estivill, H. G. Joost, W. Becker. Biochem Biophys Res Commun. 1999, 254, 474-9.

11. X. Deng, D. Z. Ewton, S. Li, A. Naqvi, S. E. Mercer, S. Landas S. Cancer Res. 2006, 66, 4149-58.

12. X. Deng, D. Z. Ewton, B. Pawlikowski, M. Maimone, E. Friedman. J Biol Chem. 2003, 278, 41347-54.

13. J. Hu, E. Friedman. Genes Cancer. 2010, 1, 803-811.

14. J. Hu, H. Deng, E.A. Friedman. Int J Cancer. 2013, 132, 2258-2269.

15. K. Jin, D. Z. Ewton, S. Park, J. Hu, E. Friedman. J Biol Chem. 2009, 284, 22916-22925.

16. K. Lee, X. Deng, E. Friedman E. Cancer Res. 2000, 60, 3631-7.

17. J. P. MacKeigan, L. O. Murphy, J. Blenis. Nat Cell Biol. 2005, 7, 591-600.

18. R. Beema Shafreen, C. Selvaraj, S. K. Singh. J. Mol. Recognit. 2013, 26, 276-285.

19. D. J. Rayalu, C. Selvaraj, S.K. Singh, R. Ganeshan, N. U. Kumar, P. Seshapani. Bioinformation. 2012, 8, 81-86.

20. H. J. C. Berendsen, D. van der Spoel, R. van Drunen. Comput Phys Commun. 1995, 91, 43-56

21. W. F. van Gunsteren, S.R. Billeter, A. A Eising, P. H. Hünenberger, P. K. H. C. Krüger, A.E. Mark, W.P.R. Scott, I.G. Tironi. 1996.

22. A.W. Schuttelkopf, D. M. F. van Aalten. Acta Crystallogr. 2004, 60, 1355-1363.

23. H. Andersen. J Chem Phys. 1980, 72, 2384-2393.

24. C. Sathishkumar, S. Gurudeeban, C. Selvaraj, A. C. Kaushik, S. Kaliamurthi, A. Khan, S. K. Singh. D. Q. C. Wei. Int J Biol Macromol. 2020, 144, 53-66.

25. C. Selvaraj, A. Omer, P. Singh, S.K. Singh. Mol Biosys. 2015, 11, 178-189.

26. C. Selvaraj, S. K. Singh. J Biomol Struct Dyn. 2014, 32, 1333-49.

27. S. K. Tripathi, C. Selvaraj, S.K. Singh, K. K. Reddy. Med Chem Res. 2012, 21, 4239-51.

28. C. Selvaraj, P. Singh, S. K. Singh. J. Mol. Recognit. 2014, 27, 696-706.

29. C. Selvaraj, P. Singh, S. K. Singh. Appl Biochem Biotechnol. 2014, 172, 1790-806.

30. C. Selvaraj, G. Krishnasamy, S. S. Jagtap, S.K. S. Patel, S.S. Dhiman, T.S. Kim, S. K. Singh, J. K. Lee. Biochem Eng J. 2016, 114, 244-256.

31. V. Ramar, S. Pappu. Comput Biol Chem. 2016, 62, 29-35.

32. R. Vanajothi, V. Hemamalini, J. Jeyakanthan, K. Premkumar. J Biomol Struct Dyn. 2020, 38, 2800-2808.

33. M. A. Martí-Renom, A. C. Stuart, A. Fiser, R. Sánchez, F. Melo, A. Sali. Annu Rev Biophys Biomol Struct. 2000; 29, 291325.

34. A. R. Muralidharan, C. Selvaraj, S.K. Singh, C.A. Nelson Jesudasan, P. Geraldine, P. Thomas. Med Chem Res. 2013, 23, 2445-2455.

35. M.H. Urf Turabe Fazil, Sunil Kumar, S. R. Naidu, C. Selvaraj, S. K. Singh, H. P. Pandey, D. V. Singh. J Biomol Struct Dyn. 2012, 30, 74-584.

36. D. Shankaracharya Das, A. S. Vidhyarthi. Nat. Proceedings. 2011.

37. J. U. Bowie, R. Lüthy, D.A. Eisenberg. Science. 1991, 12, 164-70.

38. R. Luthy, J. U. Bowie, D. Eisenberg. Nature. 1992, 356, 83-85.

Page 9/17 
39. M. Wiederstein, M. J. Sippl. Nucleic Acids Res. 2007, 35, W407-W410.

40. C. Selvaraj, P. Singh, S. K. Singh. J Recep Sig Transd. 2014, 34, 361-371.

41. R. Vanajothi, V. Hemamalini, N.M. Al-Ansari, L. A. Al-Humaid, K. Premkumar. J Biomol Struct Dyn. 2020.

42. C. Selvaraj, P. Umesh, D.D. Chandrasekaran, B. Evzen, P. Singh, V. K. Dubey, S.K. Front Chem. 2021, 8, 1179.

43. C. Selvaraj, S. Singh, S. K. Tripathi, K. K. Reddy, M. Rama. Med Chem Res. 2012, 21, 4060-4068.

44. R. A. Friesner, J. L. Banks, R.B. Murphy, T.A. Halgren, J. J. Klicic, D. T. Mainz, P. S. Shenkin. J. Med Chem. 2004, 47, 1739-1749.

45. W. Becker. Cell Cycle. 2012, 11, 3389-3394.

46. E. Friedman. Int J Mol Sci. 2013, 14, 5560-5575.

\section{Tables}

Table 1; pharmacokinetic properties of top-ranked screened compounds

\begin{tabular}{|llllllllllll|}
\hline Compound & QPPMDCK & $\begin{array}{l}\text { QPlog } \\
\text { HERG }\end{array}$ & QPPCaco & $\begin{array}{l}\text { QPlog } \\
\text { BB }\end{array}$ & $\begin{array}{l}\text { QPlog } \\
\text { S }\end{array}$ & $\begin{array}{l}\text { QPlog } \\
\text { Po/w }\end{array}$ & MW & $\begin{array}{l}\text { Stars } \\
\begin{array}{l}\text { Rule } \\
\text { of } \\
\text { five }\end{array}\end{array} \begin{array}{l}\text { \% of oral } \\
\text { absorbance }\end{array}$ \\
\hline 7768949 & 2338.14 & -5.68 & 4207.72 & 0.018 & -4.13 & 3.63 & 237.30 & 0 & 0 & 100 \\
\hline 7771055 & 262.87 & -6.65 & 262.54 & -1.611 & -5.91 & 3.19 & 428.48 & 0 & 0 & 88.92 \\
\hline 7758866 & 500.97 & -5.62 & 1011.71 & -0.725 & -4.24 & 2.58 & 312.32 & 0 & 0 & 95.88 \\
\hline 7764195 & 828.28 & -6.34 & 659.66 & -0.699 & -5.95 & 3.96 & 371.84 & 0 & 0 & 100 \\
\hline
\end{tabular}

Table 2: Scoring values of top-ranked lead molecules from virtual screening.

\begin{tabular}{|llllll|}
\hline S. No & Compound ID & Docking score Kcal/mol & Glide energy Kcal/mol & $\begin{array}{l}\text { Prime MM-GBSA } \\
\Delta \text { H-bond }\end{array}$ & (Kcal/mol) \\
\hline 1 & 7768949 & -11.361 & & -102.21 & Leu83 (2) \\
\hline 2 & 7771055 & -10.532 & -34.335 & & Ile7 \\
\hline 3 & 7758866 & -10.318 & -49.771 & -96.04 & Leu83 (2) \\
\hline 4 & & & & & Asn86 \\
\hline & & & -39.206 & -98.12 & Leu83 (2) \\
\hline
\end{tabular}

Yang C, Ji D, Weinstein EJ, Choy E, Hornicek FJ, Wood KB, Liu X, Mankin H, Duan Z: The kinase Mirk is a potential therapeutic target in osteosarcoma. Carcinogenesis. 2012, 31: 552-558.

\section{Figures}




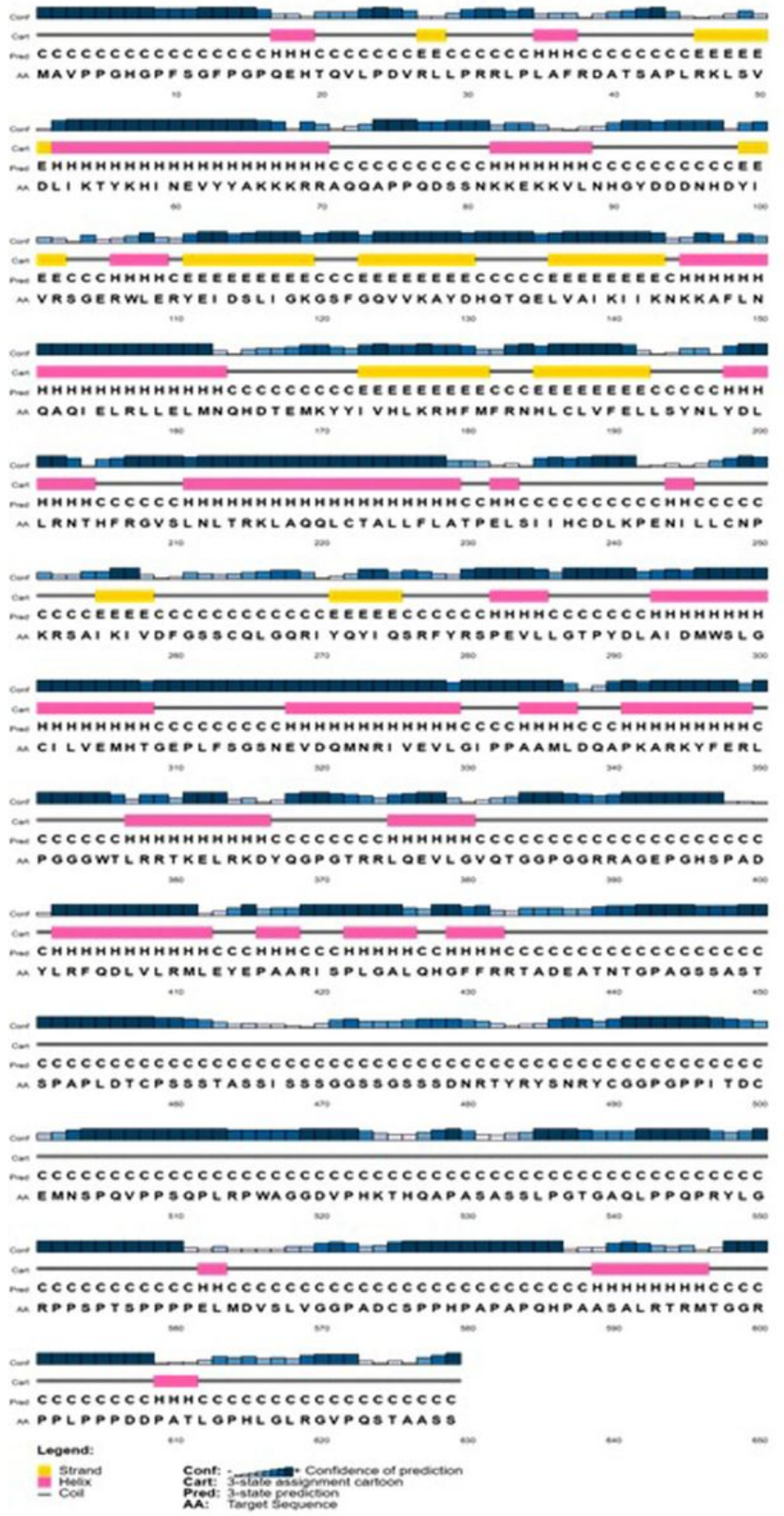

\section{Figure 1}

Secondary structure prediction and sequence alignment of modeled Mirk protein with its template protein sequence. 


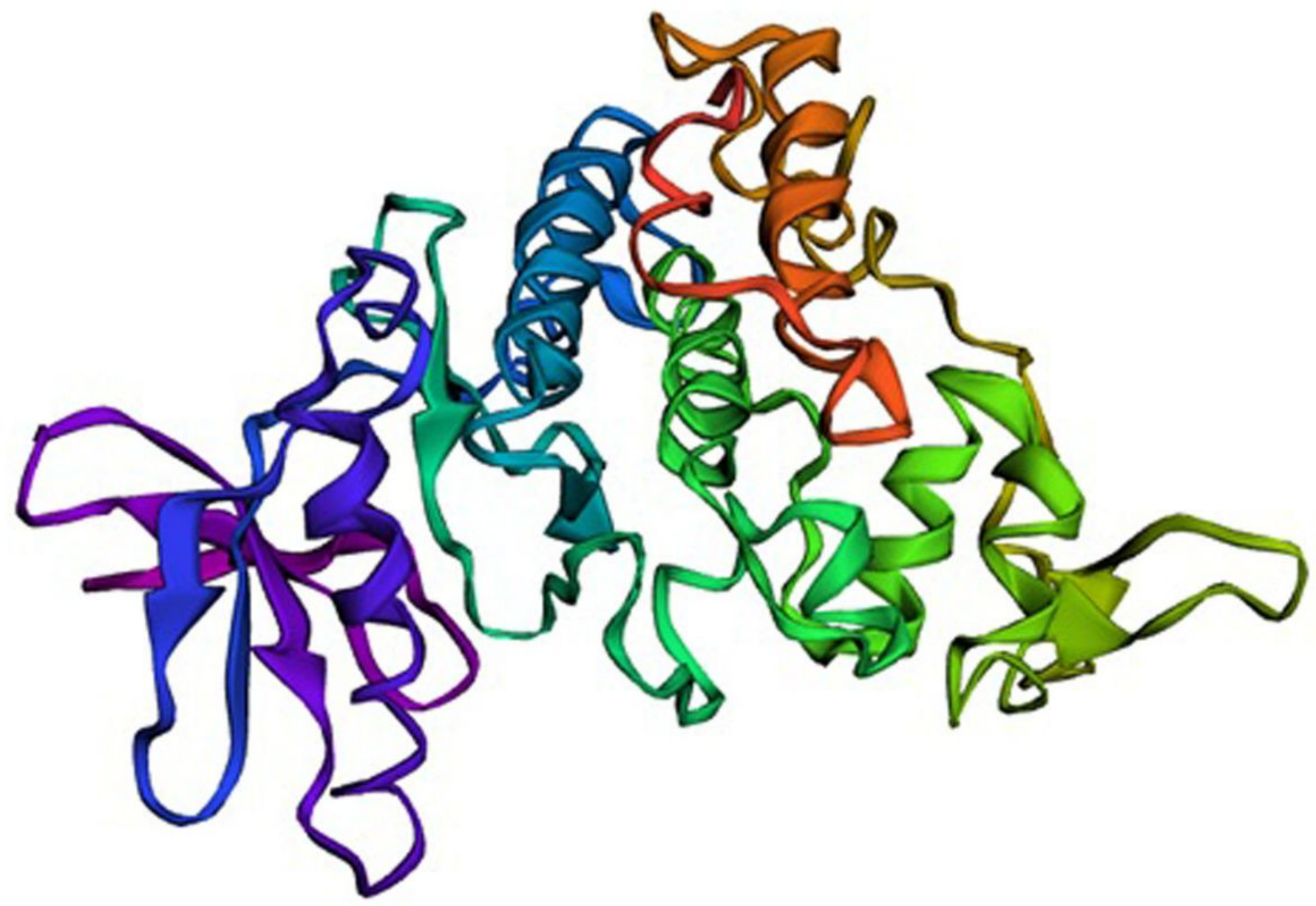

Figure 2

Modeled 3-dimensional structure of modeled Mirk protein.

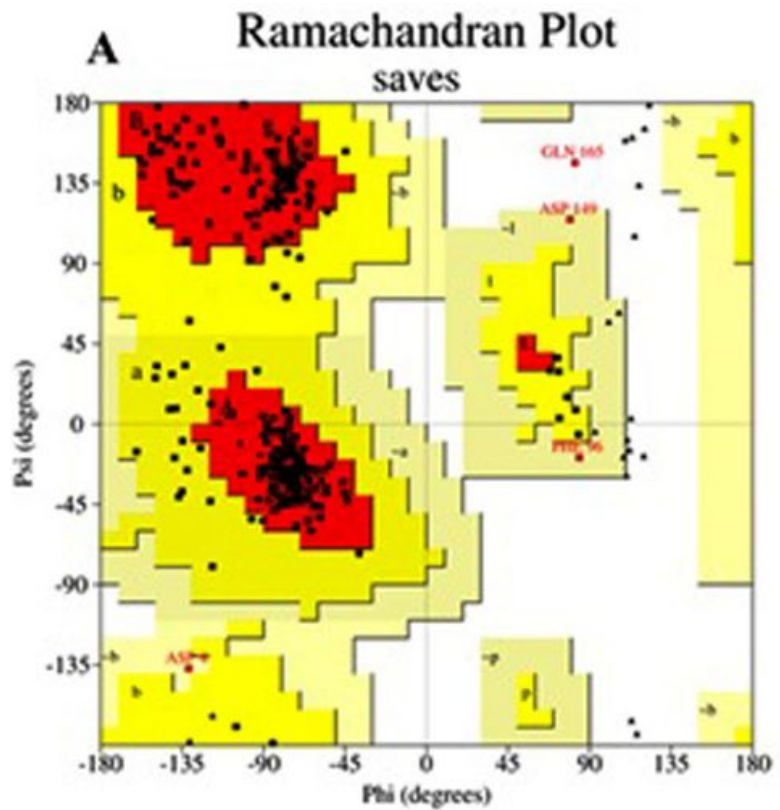

B

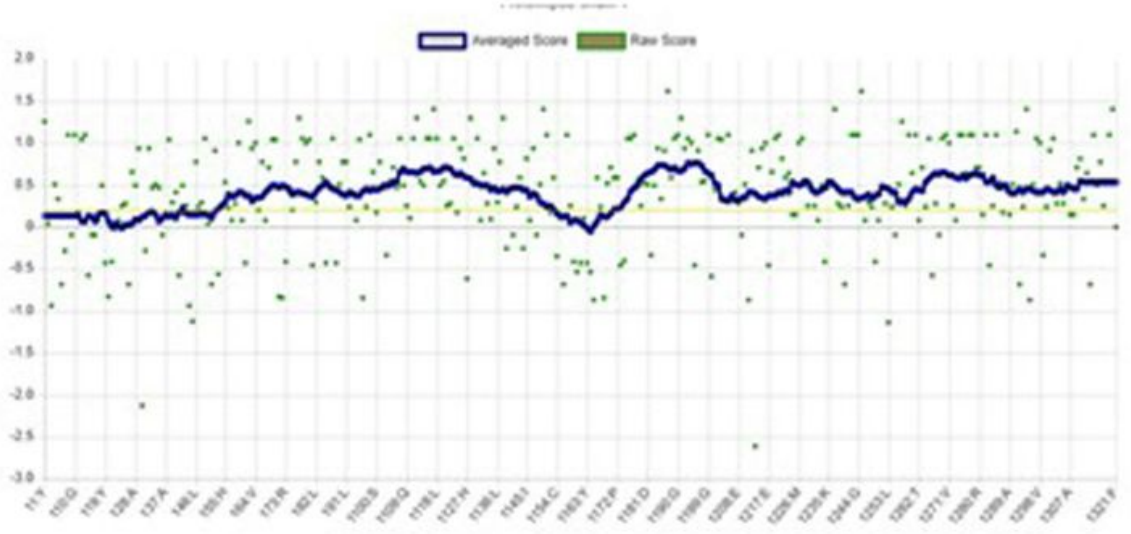




\section{Figure 3}

(A) Ramachandran plot of Mirk model generated by Modeler software; (B) Quality verification plot of modeled structure performed using Verify-3D.

$\mathbf{A}$

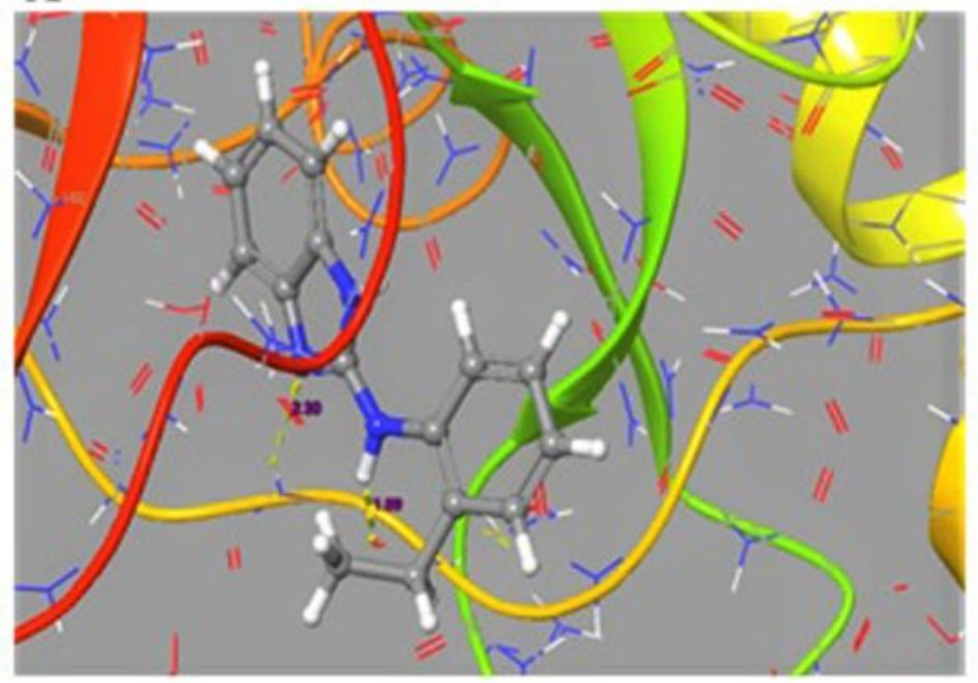

C

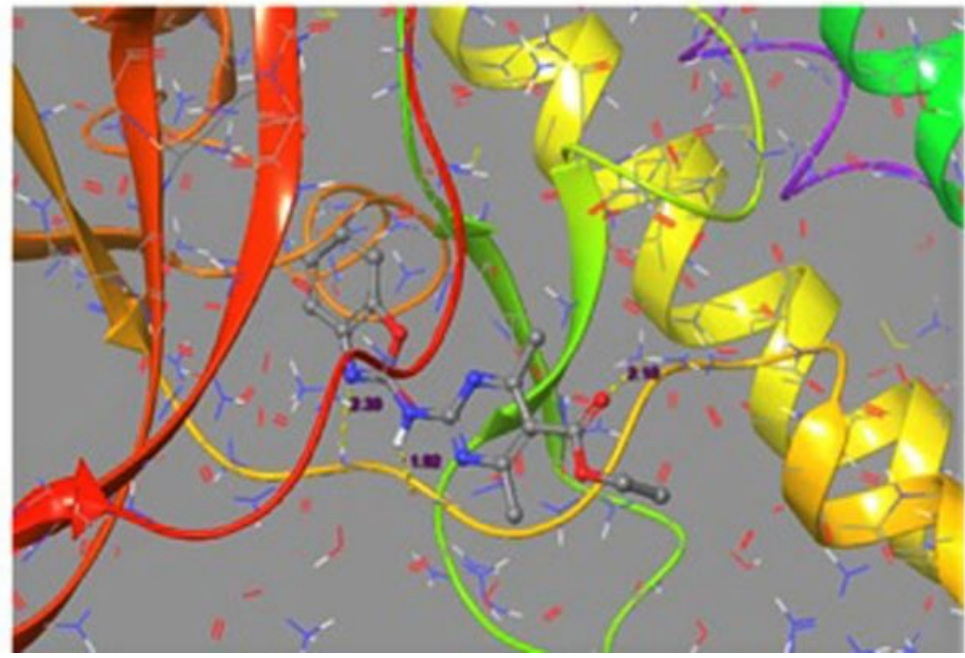

B

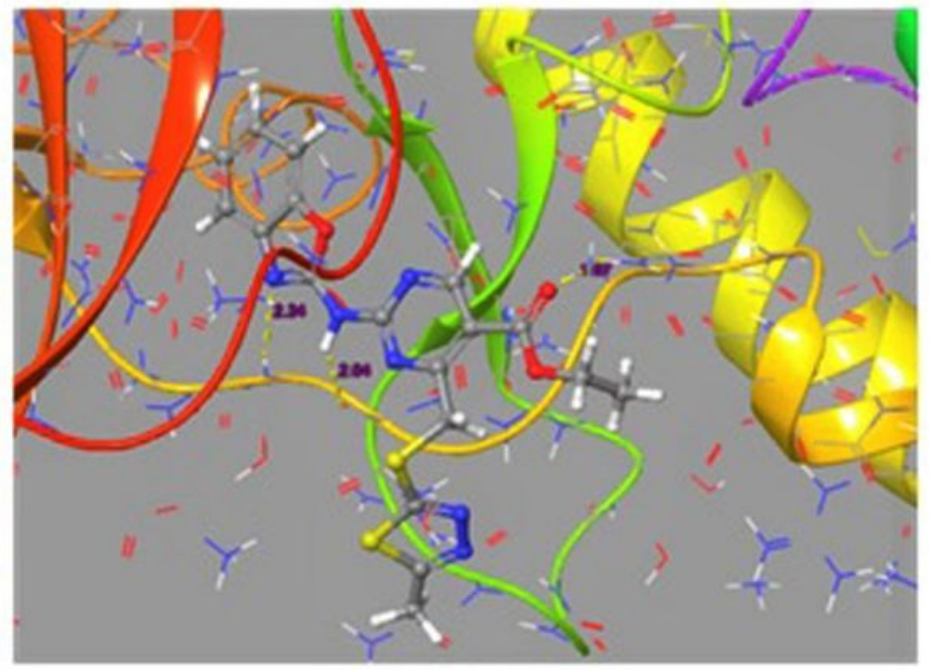

D

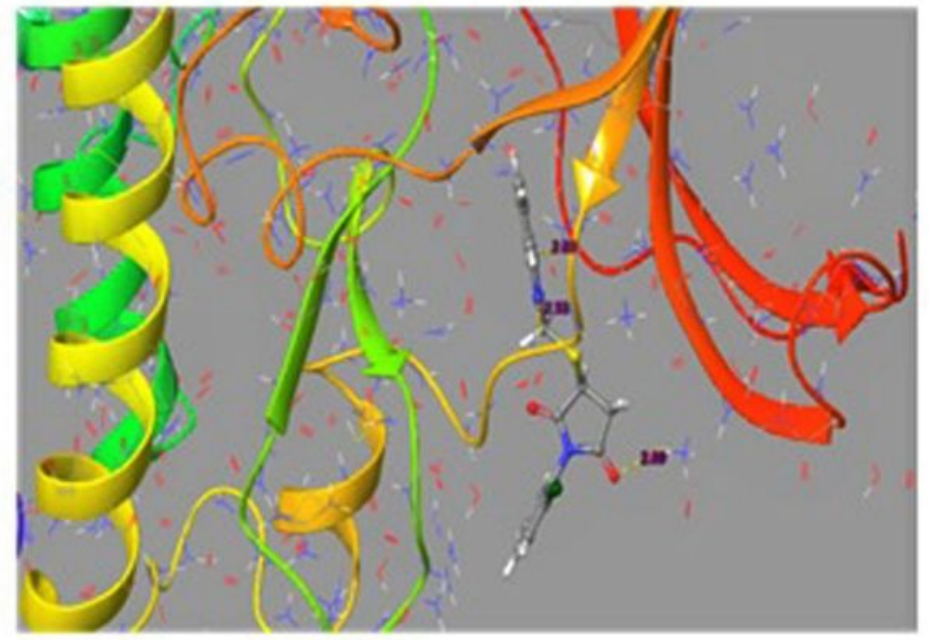

\section{Figure 4}

Interaction of top-ranked molecules from virtual screening with Mirk. (A) Interaction of Chembridge ID: 7768949 with Mirk active site residues; (B) interaction of Chembridge ID: 7771055 with Mirk active site residues; (C) interaction of Chembridge ID: 7758866 with Mirk active site residues and (D) interaction of Chembridge ID: 7764195 with Mirk active site residues. 

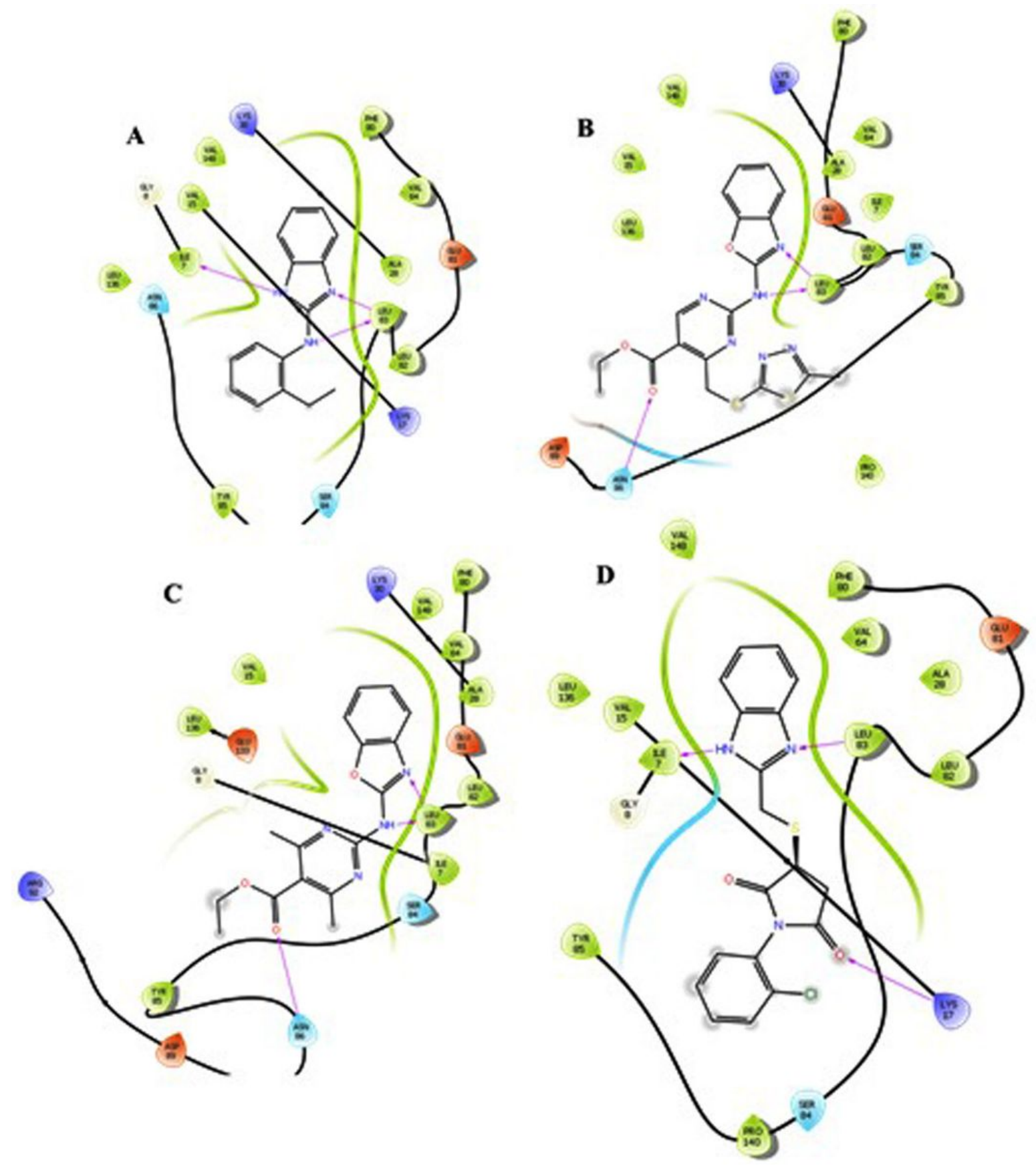

Figure 5

2D interaction of top-ranked molecules from virtual screening with Mirk. (A) Interaction of Chembridge ID: 7768949 with Mirk active site residues; (B) interaction of Chembridge ID: 7771055 with Mirk active site residues; (C) interaction of Chembridge ID: 7758866 with Mirk active site residues and (D) interaction of Chembridge ID: 7764195 with Mirk active site residues. 


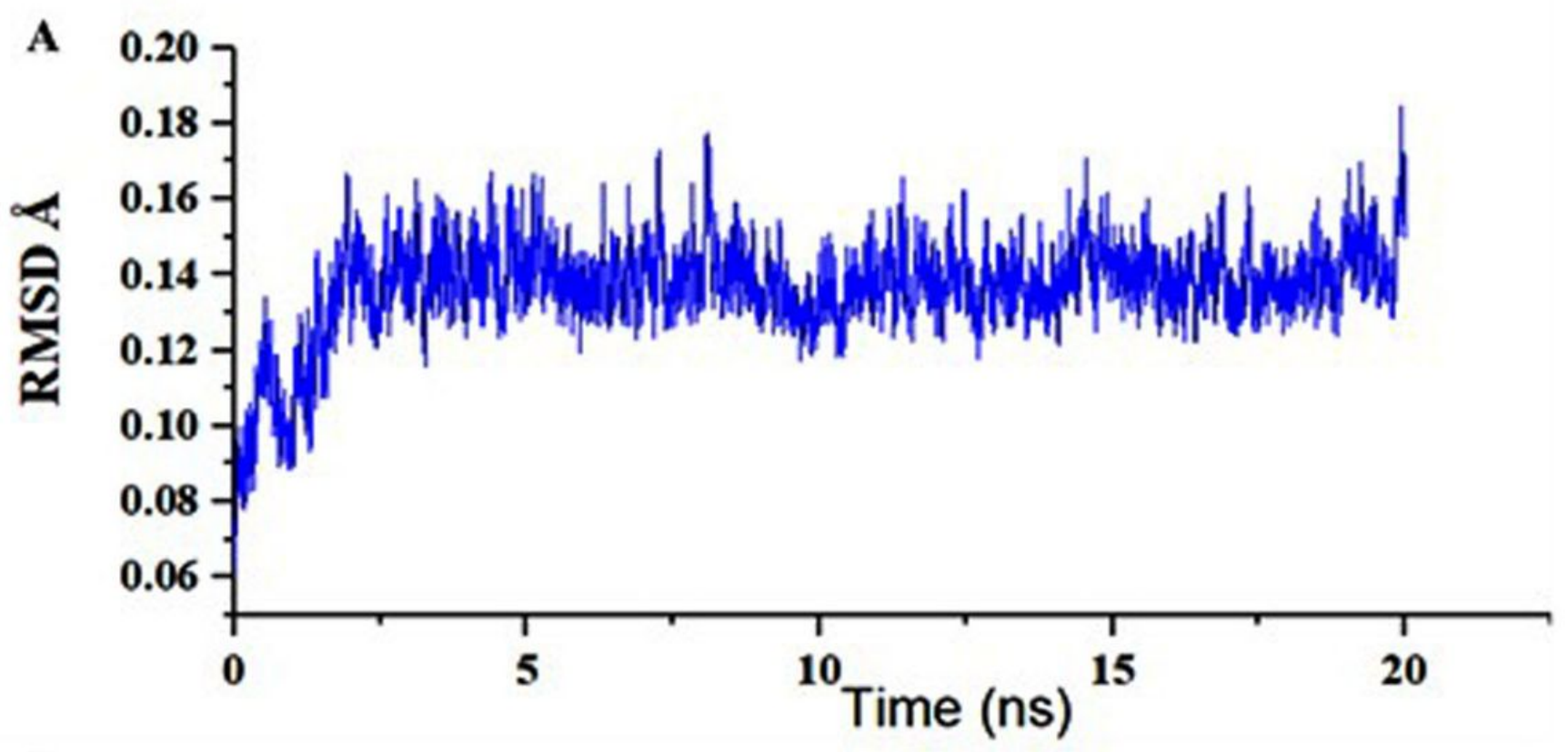

B

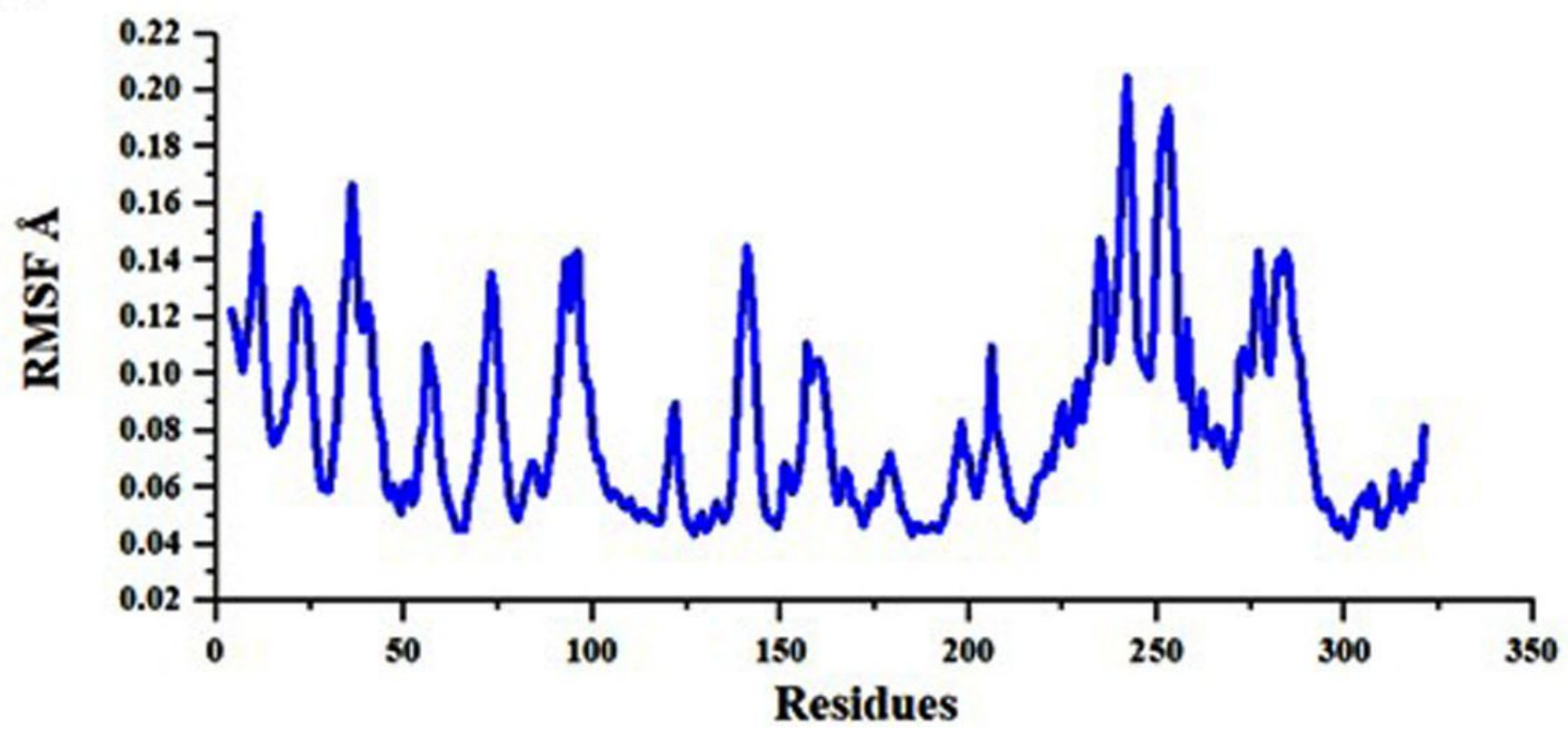

Figure 6

Molecular dynamics results. A), Root mean square deviation plot of Modeled Mirk protein and B) Root mean square fluctuation plot of apoprotein (Model structure of Mirk). 

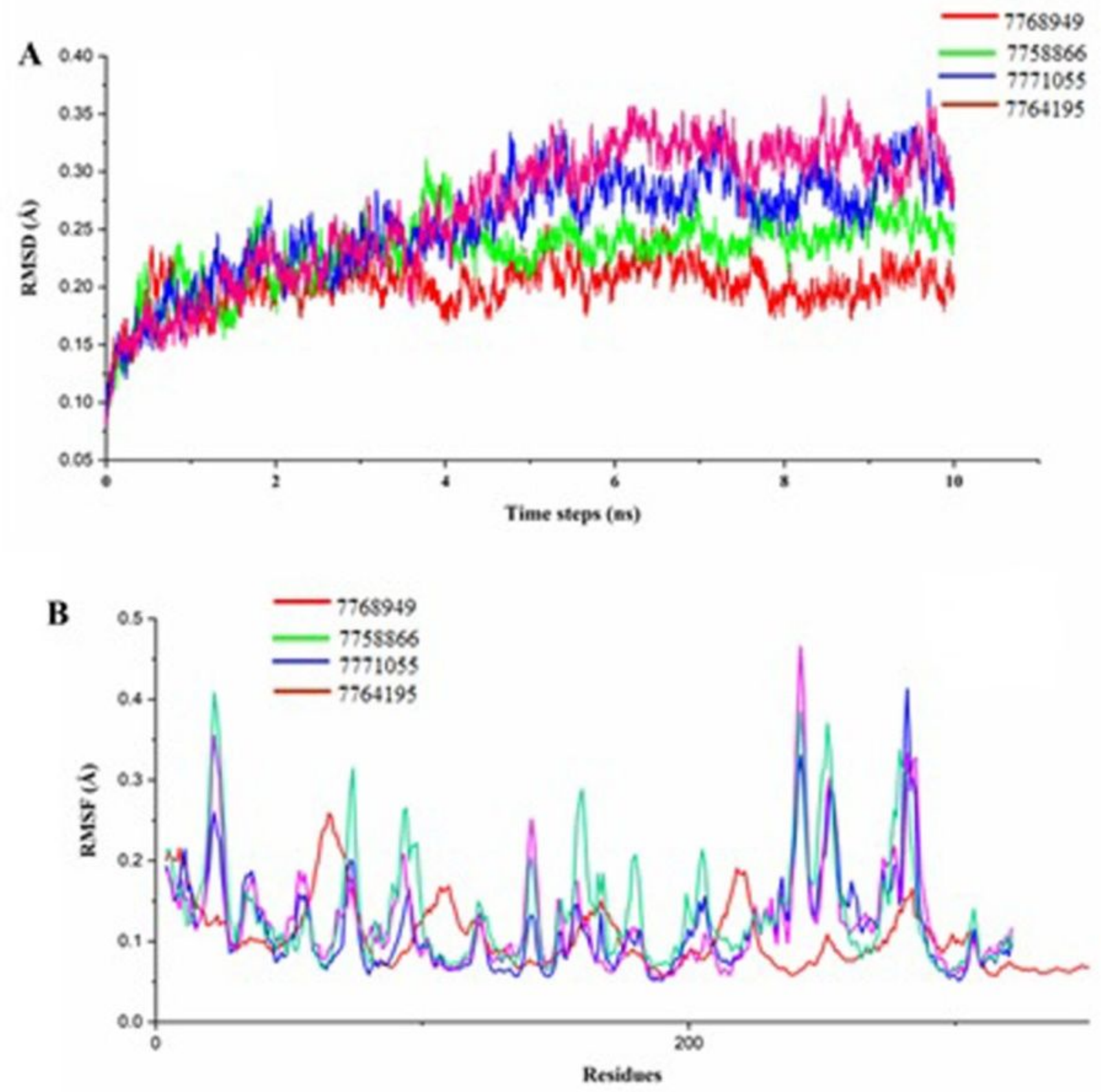

Figure 7

Molecular dynamics results. A), Root mean square deviation plot of complexes of top-ranked molecules with Mirk protein (red color represent complex with Chembridge ID: 7768949; green color represent complex with Chembridge ID: 7771055, blue color represent complex with Chembridge ID: 7758866 and purple color represent the complex with Chembridge ID: 7764195) and B) Root mean square fluctuation plot of complexes of top-ranked molecules with Mirk protein (red color represent complex with Chembridge ID: 7768949; green color represent complex with Chembridge ID: 7771055, blue color represent complex with Chembridge ID: 7758866 and purple color represent the complex with Chembridge ID: 7764195). 

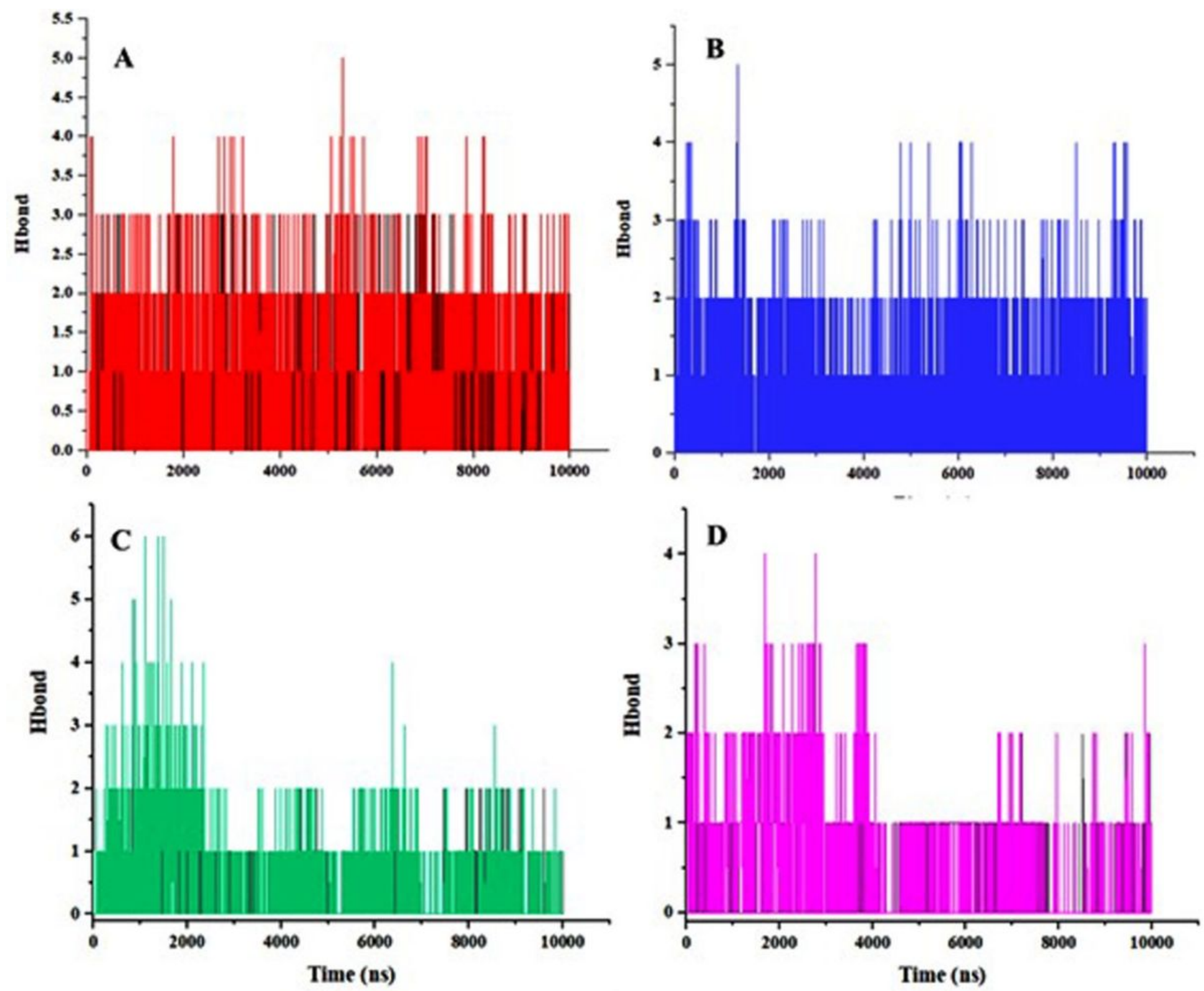

Figure 8

$\mathrm{H}$-bond interaction top-ranked molecules and their stability with active residues during $10 \mathrm{~ns}$ MD simulation (A), red color represent complex with Chembridge ID: 7768949; (B), the blue color represents complex with Chembridge ID: 7771055, (C), green color represents complex with Chembridge ID: 7758866 and (D), pink color represents the complex with Chembridge ID: 7764195. 